\title{
Neonatal Cystitis-Induced Colonic Hypersensitivity in Adult Rats: A Model of Viscero-Visceral Convergence
}

\author{
Adrian Miranda ${ }^{1}$, Aaron Mickle ${ }^{1}$, Jamie Schmidt ${ }^{2}$, Zhihong Zhang ${ }^{2}$, Reza Shaker ${ }^{2}$, Banani \\ Banerjee $^{2}$, and Jyoti N. Sengupta ${ }^{1,2}$ \\ ${ }^{1}$ Division of Gastroenterology, Department of Pediatrics, , Medical College of Wisconsin, \\ Milwaukee, Wisconsin 53226 \\ 2 Division of Gastroenterology and Hepatology, Department of Medicine, Medical College of \\ Wisconsin, Milwaukee, Wisconsin 53226
}

\begin{abstract}
Background-The objective of this study was to determine if neonatal cystitis alters colonic sensitivity later in life and to investigate the role of peripheral mechanisms.

Methods-Neonatal rats received intravesical zymosan, normal saline, or anesthesia only for three consecutive days (postnatal days 14th-16th). The estrous cycle phase was determined prior to recording the visceromotor response (VMR) to colorectal distension (CRD) in adult rats.

Eosinophils and mast cells were examined from colon and bladder tissue. CRD or urinary bladder distension (UBD)-sensitive pelvic nerve afferents (PNAs) were identified and their responses to distension were examined. The relative expression of N-methyl-D-aspartic acid (NMDA) NR1 subunit in the $\mathrm{L}_{6}-\mathrm{S}_{1}$ spinal cord was examined using Western blot.
\end{abstract}

Results-The VMR to CRD $(\geq 10 \mathrm{mmHg})$ in the neonatal zymosan group was significantly higher than control in both the diestrus, estrus phase and in all phases combined. There was no difference in the total number of eosinophils, mast cells or number of degranulated mast cells between groups. The spontaneous firing of UBD, but not CRD-sensitive PNAs from the zymosan rats was significantly higher than the control. However, the mechanosensitive properties of PNAs to CRD or UBD were no different between groups ( $p>0.05$ ). The expression of spinal NR1 subunit was significantly higher in zymosan-treated rats compared to saline treated rats $(\mathrm{p}<0.05)$.

Conclusion-Neonatal cystitis results in colonic hypersensitivity in adult rats without changing tissue histology or the mechanosensitive properties of CRD-sensitive PNAs. Neonatal cystitis does results in overexpression of spinal NR1 subunit in adult rats.

\section{Keywords}

cystitis; visceral hyperalgesia; neonatal; viscero-visceral convergence

Address for correspondence: Adrian Miranda M.D., Division of Gastroenterology and Hepatology, Medical College of Wisconsin, 8701 Watertown Plank Road, Milwaukee, WI 53226, Tel: 414-456-4011, Fax: 414-456-6361, amiranda@mcw.edu.

Contributions to Manuscripts:

Adrian Miranda: Contributed with research design, performing research, data analysis and manuscript writing.

Aaron Mickle: Performed experiments and analyzed the data.

Jamie Schmidt: Performed histological experiments and analyzed the data.

Zhihong Zhang: Performed some of the electrophysiology experiments.

Reza Shaker: Helped with designing research study and with manuscript preparation.

Banani Banerjee: Contributed essential reagents and performed histological experiments.

Jyoti N. Sengupta: Contributed with research design, performing research, data analysis and manuscript writing.

No conflicts of interest exist with any of the authors. 


\section{Introduction}

Clinical observations in patients with painful functional bowel disorders such as irritable bowel syndrome (IBS) or functional abdominal pain suggest an impact of adverse early life events on the development of visceral hypersensitivity later in life (1). A recent study reported that recurrent urinary tract infections (UTIs) during childhood correlate with the development of chronic pelvic pain, a condition that often overlaps with IBS (2). A population based study demonstrated that abdominal pain in children has a weekly prevalence of 38\%, the majority of which are females (3). Interestingly, the prevalence rates of febrile UTIs in female children have been shown to be as high as $8.3 \%(4,5)$. Spinal neurons in the lumbo-sacral and thoraco-lumbar spinal cord receive convergent inputs from both the urinary bladder and the colon (viscero-visceral convergence), suggesting that an insult to one visceral organ could lead to hypersensitivity in another pelvic structure (6). Such convergence may explain why $40-60 \%$ of patients diagnosed with IBS also exhibit symptoms and fulfill the diagnostic criteria for interstitial cystitis $(7,8)$.

We have previously reported a neonatal model of viscero-somatic convergence related visceral hyperalgesia. Acidic saline injections into the gastrocnemius muscle during postnatal days 12-20 sensitizes spinal neurons and results in chronic visceral hyperalgesia that is prevented by NMDA receptor antagonists (9). Previous studies suggest that spinal NMDA receptors contribute to dorsal horn neuron hyperexcitability and chronic hyperalgesia (1013). While the NMDA receptor is composed of multiple subunits, the NR1 subunit is ubiquitously expressed in all functional NMDARs and has been shown to be up-regulated in the spinal cord in other models of visceral hyperalgesia (14).

Zymosan, a yeast cell wall derivative, is considered a "natural" inflammogen and has been recently reported to induce cystitis in neonatal rats (15). In adult female rats, bladder inflammation with zymosan acutely increases sensitivity to UBD, while in neonatal rats, inflammation with zymosan increases bladder sensitivity that persists into adulthood $(16,17)$. While the exact mechanism is not known, the role of PNAs in the development of visceral sensitivity has recently received much attention. In adult rats, colon irritation with TNBS sensitizes bladder PNAs to mechanical and chemical irritation and has also been shown to increase the percentage of activated masts cells in the bladder $(18,19)$. In adult rats, acute bladder inflammation with KCL has been shown to produces cross-sensitization of pelvic afferents and colon hypersensitivity to mechanical distension (20). It has been speculated that dichotomizing afferents play an important role in the development of the hypersensitivity (21).

We hypothesized that neonatal bladder inflammation results in cross-organ sensitization and long-term changes in colon sensitivity. We investigated whether the presence of mast cells and/or eosinophils in the urinary bladder or colon are necessary for the maintenance of colon hyperalgesia in adult rats following neonatal cystitis and also examined the response characteristics of PNAs to mechanical distension. We also examined the expression of the NR1subunit in the spinal cord to determine if spinal NMDA receptors have a potential role in the maintenance of chronic visceral hypersensitivity.

\section{Materials and Methods}

\section{Animals}

Experiments were performed on female Sprague-Dawley rats. Time pregnant female rats were obtained from Harlan (Indianapolis, IN, USA) and maintained in separate cages. Rat pups were born in our facility and only female pups were used in this study. The pups remained housed with their mothers until they were weaned at postnatal (PN) day 21. As 
adults, the rats were kept in controlled conditions with a 12 hour light/dark schedule and had access to both food and water ad libitum. The Institutional Animal Care and Use Committee at Medical College of Wisconsin approved all experimental protocols (MCW AUA 355).

\section{Neonatal Zymosan Induced Cystitis}

Neonatal rats (P14) were separated into three experimental groups: (a) intravesical zymosan, (b) saline and (c) anesthesia-only. Pups were anesthetized with halothane (5\% for induction and $2 \%$ for maintenance). A 24-gauge intravesical catheter (B-D Insyte-N Autoguard, Sandy, UT) was placed transurethrally and $0.1 \mathrm{ml}$ of $1 \%$ zymosan solution or saline was instilled into the bladder and left in place for 30 minutes while under anesthesia. At the end of the procedure, fluid was drained back into the syringe, the catheter was removed and the pup was returned to the cage. The third group simply received 30 minutes of anesthesia without any manipulation. The anesthesia only group was added in the study to test whether volume distension influenced visceral sensitivity. In all 3 groups (zymosan, saline and anesthesia only), the treatments were repeated daily for 3 days after which the rats were allowed to grow to adulthood (2-3 months) without any further interventions.

\section{Visceromotor Response (VMR)}

All surgical procedures were performed in adult rats under deep anesthesia with sodium pentobarbital (50 $\mathrm{mg} \cdot \mathrm{kg}^{-1}$ i.p.). Baytril (3mg per day, p.o.) was given postoperatively for a total of 5 days in all animals to prevent infection. Twenty-four hours prior to surgery, the animals were placed in wire bottom cages and access to food, but not water, was denied in order to empty the stomach. Teflon coated electrodes (Cooner wire, Part\#: A5631) were implanted in the external oblique muscle for EMG recordings. The pair of electrodes was externalized subcutaneously and protected using silastic tubing sutured to the dorsal aspect of the neck. Rats were housed separately following surgery and allowed to recuperate for at least 5 days prior to VMR recordings.

The VMR to CRD was quantified using EMG recordings from the external oblique muscle as an objective measure of visceral sensation in all groups as previously described (22). The rats were kept in individual Bollman cages while a distensible latex balloon $(5 \mathrm{~cm}$ in length) was inserted into the descending colon and rectum. The balloon was attached to a barostatic distension device via a polyethylene tube and was kept in place by taping the catheter to the tail. A stimulus-response function (SRF) to graded distending pressures $(10,20,30,40,50$, and $60 \mathrm{mmHg}, 30 \mathrm{~s}$ ) was carried out for each rat. The distension pressure was incremented every 3 minutes. The EMG signal from the external oblique muscle was amplified through a low noise AC differential amplifier (model-3000: A-M Systems, Inc.) and recorded on-line using the Spike 3/CED 1401 data acquisition program (CED 1401; Cambridge Electronic Design, Cambridge, UK).

\section{Estrous Cycle}

Weekly measurements were taken to identify the phases of the estrous cycle prior to assessment of visceral sensitivity in all rats. All recordings were taken from the same rats across different estrous cycles. Intact female rats were primarily classified into one of three phases; proestrus, diestrus or estrus on the day of the VMR recordings based on the cellular characteristics of the vaginal smears. Since the metestrus phase only lasts approximately 5-6 hours and previous studies have shown similar serum estrogen levels in this phase compared to the diestrus, no experiments were performed during the metestrus phase.

In order to assess the cycling pattern of each rat, vaginal smears were collected once daily between 10:00am to11:00 am immediately prior to VMR recordings. The samples were 
placed on glass slides and viewed under dark field illumination at 100X magnification to determine the phase as previously described (23).

\section{Bladder and Colon Histology}

To evaluate for any possible bladder or colon inflammation, histological examination of tissues were preformed in zymosan- $(n=4)$ and saline-treated rats $(n=4)$. Briefly, the rats were euthanized with Beuthanasia-D (Schering-Plough Animal Health, USA) (0.5ml, i.p.) and the distal colon and bladder were removed and sectioned at $10 \mu \mathrm{m}$ thickness and stained with hematoxylin and eosin. The sections were examined under light microscopy for microscopic changes in a blinded fashion. Tissue was evaluated for neutrophilic infiltrates in the lamina propria and the degree of mucosal and submucosal edema. To investigate eosinophilic counts, tissue sections (10 $\mu \mathrm{m}$ thickness) were stained with EPO using 3,3'diaminobenzidine tetrahydrochloride (DAB; Sigma). The sections were incubated initially with $10 \mathrm{mM}$ cyanide buffer and rinsed in PBS, followed by incubation with peroxidase substrate $\mathrm{DAB}$ for 10 minutes at room temperature. The slides were rinsed thoroughly with water and cover-slipped before examination under the microscope. For both zymosan- $(n=3)$ and saline-treated $(n=3)$ rats, two random slides containing four tissue sections per slide of the corresponding bladder or colon were examined for eosinophil. The results were quantified by a single, blinded investigator under high power field (20x) and averaged together for each animal.

For quantification of mast cells, colon and bladder tissue was stored overnight in $4 \%$ paraformaldehyde, the tissues were dehydrated in graded alcohol and paraffin embedded. Serial sections of $10 \mu \mathrm{m}$ thickness were cut and the slides oven-dried at $60^{\circ} \mathrm{C}$. Sections were de-paraffinized by two times washes ( 5 minutes each) with xylene followed by two times wash with $100 \%$ ethanol and a 5 minute wash in $95 \%$ ethanol and hydrated with distilled water. Slides were then placed in working Toluidine Blue (10\% Toludine Blue stock in $1 \%$ Sodium Chloride; Toluidine Blue stock: $1 \mathrm{gm}$ TBO in $100 \mathrm{ml}$ of $70 \%$ alcohol) for 1-2 minutes followed by 3 minute rinses in distilled water three times. Slides were quickly dehydrated through $95 \%$ and $100 \%$ alcohols and cleared in xylene and cover slipped. Mast cell numbers and activation were quantified by a single, blinded investigator under high power field (20X) as previously described (24). For both zymosan- $(n=6)$ and saline-treated $(n=6)$ rats, two random slides, each containing four sections per slide of the corresponding bladder or colon were scanned for intact and degranulated mast cells. The results were averaged together for each animal.

\section{Pelvic nerve afferent (PNA) fiber recordings}

The surgical procedure and recordings from PNAs in the S1 sacral dorsal root were carried out as previously described (25). Briefly, following anesthesia and preparation, the pelvic nerve afferent fibers in the sacral S1 dorsal root of zymosan- or saline-treated rats were identified by recording the evoked response to CRD $(60 \mathrm{mmHg})$ or UBD $(0.6 \mathrm{ml})$. A CRDsensitive fiber was always tested to UBD $(0.6 \mathrm{ml})$ and vice-versa to test the dichotomized innervation to two organs. Once the increase ( $20 \%$ above the spontaneous firing) in firing to distension was confirmed, a SRF was recorded to either graded CRD $(10-60 \mathrm{mmHg}, 30 \mathrm{~s})$ or to UBD $(0.1-0.8 \mathrm{ml}, 30 \mathrm{~s})$ depending on the innervation of the fiber. At the end of the experiments, the rats were euthanized by injecting a lethal dose of Beuthanasia-D $(0.2 \mathrm{ml}$, i.v.).

\section{NR1 Expression in Spinal Cord (Western Blot)}

Western blot for spinal NR1expression was carried out in adult rats that received either intravesical zymosan or saline during the neonatal period. The methods have been performed in our laboratory and previously described (26). Briefly, crude extracts from rat's 
dorsal spinal cord were prepared by powderizing the tissues in liquid nitrogen and homogenization in ice-cold hypotonic lysis buffer (10 mM Tris HCl, 5 mM EDTA, pH 8.0) containing protease inhibitor cocktail tablet (Complete Mini, Roche Diagnostics, Indianapolis, IN) and phosphatase inhibitor cocktail (Thermo Scientific, Rockford, IL), followed by differential centrifugation first at $1,200 \mathrm{rpm}$ for $30 \mathrm{~min}$ and then for another 30 $\min$ at $14,000 \mathrm{rpm}$. The membrane pellet was solubilized for $30 \mathrm{~min}$ on ice with RIPA buffer $(150 \mathrm{mM} \mathrm{NaCl}, 50 \mathrm{mM}$ Tris HCl, pH 7.5, $1 \mathrm{mM}$ EDTA, $1 \mathrm{mM}$ PMSF, $1 \%$ Triton $\mathrm{X}-1000,0.5 \% \mathrm{Na}$ deoxycholate, $0.1 \%$ SDS containing protease and phosphatase inhibitors). The membrane extracts were centrifuged and assayed for protein content by the bicinchoninic acid (BCA) method (Thermo Fisher Scientific, Rockford, IL). Approximately $25 \mu \mathrm{g}$ spinal cord tissue extracts were electrophoresed on $8 \%$ SDS-PAGE and transferred onto nitrocellulose membrane. After transfer, the membrane was blocked with 5\% nonfat milk and then probed with antibodies to NR1 subunit (mouse anti-NR1, 1:100, BD Biosciences, San Jose, CA). Protein bands were visualized by using a horseradish peroxidase conjugated secondary antibody (Jackson ImmununoResearch, West Grove, PA) and an enhanced chemiluminescent detection system (Thermo Scientific).

\section{Statistics}

Statistical analysis was performed using SigmaStat (V2.03, SPSS Inc, Chicago, IL). All data are expressed as mean \pm SEM unless otherwise stated. For VMR recordings, the area under the curve was measured from the EMG. Data were analyzed using two-way repeated measures (ANOVA) and by Student-Newman-Keuls for multiple comparisons.

For analysis of PNA fibers, the total number of action potentials over a 60 second resting period prior to colon or bladder distension and during the distension period (30 seconds) was counted and represented as impulses/sec. To measure the actual changes in response of the neurons to distension, the mean firing frequency during the resting period was subtracted from the mean firing frequency during colon distension. Neuronal firing in impulses per second was analyzed at baseline and each distention pressure using two-way repeated measures ANOVA.

Mast cell counts were performed using high-power evaluation in sections stained with toluidine blue and quantified per whole slice. For Western blot, the relative changes in the intensity of NR1 subunit expression was normalized against the intensity of housekeeping gene $\beta$-actin for the same tissue sample using alphaimager 3400 software (Cell Biosciences, Inc., Santa Clara, CA). Independent-sample t-tests were used to analyze the histological measures of mast cells, eosinophil counts and NR1 expression. In all instances, a p-value < 0.05 was considered significant.

\section{Results}

\section{Visceromotor response to CRD}

There was a significant increase in the VMR to CRD at distension pressures $\geq 10 \mathrm{mmHg}$ in the neonatal zymosan-treated group in both the diestrus and estrus phase when compared to either anesthesia or saline groups (fig. 1B and 1C). Since CRD pressures $<30 \mathrm{mmHg}$ are not considered noxious in rats and humans, the visceral organ cross-sensitivity induced via neonatal cystitis has characteristics of both allodynia and hyperalgesia. While it is well established that the proestrus phase (when plasma estrogen concentrations are highest) is associated with a higher magnitude of response to CRD in rats (27), there was no difference in the response to CRD between groups in this phase at all distension tested (fig. 1A). It was also noted that the responses of the control groups (saline and anesthesia) to CRD were higher during the proestrus phase. Post-hoc analysis revealed that the VMR of saline and 
anesthesia control groups in the proestrus phase tended to be higher at CRD pressures greater that $30 \mathrm{mmHg}$ compared to estrus or diestrus phase ( $p>0.05$ ). The increased sensitivity of the control rats during the proestrus phase likely influenced the comparison to the zymosan treated group. To evaluate the overall effect of the estrous cycle on the observed hypersensitivity, the data from all phases of the estrous cycle was pooled and analyzed. A significant increase in the VMR was observed in the zymosan-treated group irrespective of the phase of the estrous cycle compared to saline or anesthesia only rats at distension pressures $\geq 10 \mathrm{mmHg}$ (Figure 1D). Since the VMR of saline treated rats did not differ from that of anesthesia only rats, we excluded the anesthesia only group from subsequent experiments.

\section{Histology}

All microscopic evaluations of bladder and colon tissue were performed in adult rats that received either zymosan or saline during the neonatal period. Examination of these tissues following H\&E staining did not reveal any damage to the epithelium or significant polymophonuclear (PMN) cell infiltration across groups (fig 2A-D). Eosinophils were quantified using EPO staining on urinary bladder and colon tissue and representative samples are shown in figure 3(A-D). Overall, there was no difference in the total number of eosinophils in either bladder or colon tissue between groups (Figure 3E). In order to investigate the role of mast cells in the maintenance of chronic visceral hyperalgesia in this model, the total number of mast cells, as well as degranulated mast cells, was calculated in both bladder and colon tissue and compared between groups. Representative photomicrographs are shown in figure 4(A-D). The average number of mast cells present in the colon and bladder of neonatal zymosan rats was no different than that of control (4E). Comparison between groups also demonstrated no difference in the number of degranulated mast cells found in both colon and bladder sections (4F).

\section{Pelvic recordings}

In order to investigate the contribution of primary sensory afferents on the observed colon hyperalgesia, we examined the mechanotransduction properties of PNA fibers from zymosan-treated rats and compared them to that of the saline-treated control. A total of 52 mechanosensitive pelvic nerve fibers from 25 rats were identified that responded to either CRD ( $n=14$ zymosan, $n=11$ saline) and UBD ( $n=16$ zymosan, $n=11$ saline). No PNA fibers that responded to both CRD and UBD were identified during recordings. In all groups, pelvic fibers exhibited spontaneous firing and an intensity-dependent increase in response to either colon or bladder distension. The spontaneous firing of CRD-sensitive PNA fibers from adult rats in the zymosan group $(1.23 \pm 0.57 \mathrm{impulses} / \mathrm{sec})$ was no different from the saline control $(1.11 \pm 0.3 \mathrm{impulses} / \mathrm{sec})(\mathrm{p}=0.68)$. However, the spontaneous firing of UBDsensitive PNA fibers from the zymosan rats was significantly higher than the saline controls ( $0.84 \pm 0.21$ vs $0.39 \pm 0.14 \mathrm{impulses} / \mathrm{sec}$, respectively) ( $\mathrm{p}=0.01)$. The responses of PNA fibers from the neonatal cystitis rats to graded CRD were no different from the control rats at all pressures tested (fig. 5C, p>0.05). Similarly, we found no difference in UBD-sensitive fiber's response to distension between groups (Fig 5D, $\mathrm{p}>0.05$ ).

\section{Spinal NR1 Expression}

Protein extracts from the L6-S1 spinal cord segments were analyzed using rat specific NR1 antibody. We have used a monoclonal antibody raised against the NMDA-NR1 subunit and generated by using a fusion protein encoding NMDA-NR1 amino acid residues 660-811, representing the intracellular loop between the transmembrane region III and IV not encompassing any splice variant regions. A specific polypeptide band of $120 \mathrm{kDa}$ was identified in the tissue extracts from both saline and zymosan-treated animals (Fig 6A). The 
relative intensity of staining against $\beta$-actin exhibited a significant increase in spinal NR1 subunit expression for zymosan treated rats compared to saline control (Fig. 6B and $\mathrm{p}<0.05$ ).

\section{Discussion}

In the current study, intravesical zymosan in female rats during the neonatal period (P14P16) was used as a model of early cystitis to investigate the long-term, cross-organ sensitization that may ultimately be involved in colon hypersensitivity and potentially explain the impact of chronic bladder pain/injury on the developing nociceptive circuits. Our study showed that: 1) neonatal cystitis results in colon hypersensitivity during adulthood that was manifested as an increase in the VMR to mechanical distension of the colon, 2) the hypersensitivity is independent of the estrous phase, 3) the hypersensitivity is maintained despite the absence of inflammation in bladder and colon tissues, 4) neonatal cystitis does not chronically sensitize PNA fibers innervating the colon or bladder to visceral distension, and 5) neonatal cystitis increases expression of the NMDA NR1 subunit in the spinal cord of adult rats.

We considered whether the phase of the estrous cycle in female rats would impact the colon sensitivity and thus we examined this for every rat prior to VMR testing. During the estrus and diestrus phase, neonatally zymosan-treated rats exhibited significant hyperalgesia compare to saline-treated and anesthesia control rats. Combining all phases of the cycle, zymosan-treated rats were persistently hyperalgesic compared to the other groups. However, during the proestrus phase, all groups of rats exhibited greater response to CRD and there was no difference between groups. This was likely due to the increased VMR of control animals during the proestrus phase, the period when all rats are known to be hypersensitive (27). In other words, control animals displayed a higher response to CRD during this phase while the neonatal cystitis group reached a peak in response that made the difference negligible.

Since previous studies have suggested a link between activated mast cells and bladder hypersensitivity, we examined whether this cross-organ sensitization is associated with chronic tissue inflammation and/or mast cell degranulation in the bladder or colon (28). However, histological examination of both organs failed to show and evidence of chronic inflammation, increased eosinophils, mast cell or mast cell degranulation in adult rats. Our results coincide with a recent study of neonatal zymosan induced cystitis that showed no increase in total mast cell counts or inflammation in the urinary bladder of adult rats (29). We also found no difference in the number of eosinophils present in either the bladder or colon of zymosan-treated rats compared to saline control. This is an important finding since it likely suggests a significant difference in the mechanism underlying visceral hypersensitivity following adult bladder inflammation versus inflammation that occurs during the neonatal period. It should also be noted that a previous study in rats demonstrated that following acute inflammation, the bladder is the most vulnerable to cross-organ effects and the least effective in inducing them (30). In that study, almost no cross-organ inflammation or increased vascular permeability was seen in the colon following bladder inflammation, which coincides with our findings.

Several mechanisms that could results in the observed cross-sensitization have been proposed; amongst these is the notion of sensitization of primary sensory afferents. The large bowel and urinary bladder of the rat are dually innervated by the pelvic (parasympathetic) nerves, which project to the lumbo-sacral (L6-S1) spinal segments. These afferents are generally pseudounipolar cells with their cell bodies located in the spinal dorsal root ganglia (DRG) (31). Some investigators have proposed that the viscero-visceral convergence mostly between bladder and colon could develop through dichotomizing pelvic 
afferents whereby a noxious stimulus to one organ results in neurogenic inflammation and overlapping pain to another organ via individual afferent terminals that supply two organs (pre-spinal convergence) (20). While recent retrograde tracing studies report the existence of $\sim 15 \%$ dichotomizing afferents innervating the bladder and colon (i.e., peripheral axon collaterals with one soma in DRG) in Sprague-Dawley rats, in our electrophysiology recordings, no PNAs were identified that responded to both UBD and CRD (21). To further explore other peripheral mechanisms that could explain the colon hypersensitivity, we focused on the functional properties of PNA fibers innervating the colon and urinary bladder.

Previous studies have investigated the contribution of peripheral sensitization in UBDsensitive PNA fibers following inflammation of the colon $(18,28)$. Interestingly, the current study demonstrated an increase in the baseline spontaneous firing of bladder PNA, but not colon. This is an important finding since the increase in firing could influence convergent neurons and more importantly contribute to spinal sensitization of UBD-sensitive spinal neurons that was observed in a previous study (32). In the current study, however, we did not find any enhanced response of PNAs to CRD or UBD. Thus, the mechanism responsible for maintaining the chronic visceral hypersensitivity is more likely mediated through a spinal and/or supraspinal pathway. Neonatal bladder inflammation with zymosan has already been demonstrated to increase bladder sensitivity when tested months after the initial inflammation (17). Subsequent studies have shown that naloxone (an opioid receptor antagonist) has no effect on the VMR to bladder distension in adult rats that had experienced neonatal cystitis, suggesting that the observed hypersensitivity could result from a decrease in the tonic inhibitory effect of the central opioidergic system (33). This observation supports the notion that modifications or permanent alterations can occur in supraspinal areas involved in descending inhibitory control and/or the descending facilitatory systems that are immature during the development. Thus, persistent noxious sensory inputs during infancy may not allow for these immature systems to properly develop, altering their effectiveness later in life (34). Alternatively, colon hypersensitivity following early cystitis could also result from spinal sensitization since it is known that convergence of afferent input from both bladder and colon is a common feature of interneurons in the sacral spinal cord (31). Approximately $32 \%$ of spinal neurons in the L6-S1 spinal cord receive convergent inputs from both the urinary bladder and the colon (35). In other words, an initial event that sensitizes spinal neurons can result in increased neuronal spontaneous activity, decreased firing thresholds and enhanced neurotransmission from the same organ, from other visceral structures or from somatic areas $(9,36)$. A recent study has already demonstrated an alteration in the phenotypic responses of type I spinal neurons in adult rats that had been exposed to intravesical zymosan during the neonatal period (32). This phenotypic change appears to be dependent on the "early life" insult since three consecutive days of intravesical zymosan in adolescence does not alter pain sensitivity (33). The current study also demonstrated an alteration in spinal NMDA NR1 subunit expression of adult rats that were treated with zymosan during the neonatal period. NR1 subunit upregulation may play an important role in the maintenance of chronic visceral hypersensitivity, since the upregulation was observed more than 30 days following cystitis. It has been suggested that increased expression of the NR1 subunits enhances current flow through NMDA receptors and therefore is likely to be among one of multiple factors involved in spinal sensitization (37-39). However, further studies are needed to understand the intricate mechanism.

In summary, the present study examined the effects of neonatal cystitis on colon sensitivity later in life. An effect was noted on the VMR to colorectal distension, suggesting the development of colon hypersensitivity and providing a model of viscero-visceral convergence. Our results, along with the work of others, suggests that the underlying mechanism responsible for maintaining the colonic hypersensitivity is more likely to involve 
either spinal or supraspinal pathways and that spinal NR1 expression may play an important role in mediating chronic visceral hypersensitivity.

\section{Acknowledgments}

Grant support: This work has been supported NIH K08 DK076198-01A1 grant awarded to Dr. Adrian Miranda and NIH 1R56DK089493-01 awarded to Drs. J. N. Sengupta and B. Banerjee.

\section{References}

1. Chitkara DK, van Tilburg MA, Blois-Martin N, Whitehead WE. Early life risk factors that contribute to irritable bowel syndrome in adults: a systematic review. Am J Gastroenterol. 2008; 103:765-74. Review. [PubMed: 18177446]

2. Peters KM, Killinger KA, Ibrahim IA. Childhood symptoms and events in women with interstitial cystitis/painful bladder syndrome. Urology. 2009; 73:258-62. [PubMed: 19036420]

3. Saps M, Seshadri R, Sztainberg M, Schaffer G, Marshall BM, Di Lorenzo C. A prospective schoolbased study of abdominal pain and other common somatic complaints in children. J Pediatr. 2009; 154:322-6. [PubMed: 19038403]

4. Shaikh N, Morone NE, Bost JE, Farrell MH. Prevalence of urinary tract infection in childhood: a meta-analysis. Pediatr Infect Dis J. 2008; 27:302-8. [PubMed: 18316994]

5. Freedman AL. Urologic Diseases in America Project. Urologic diseases in North America Project: trends in resource utilization for urinary tract infections in children. J Urol. 2005; 173:949-54. [PubMed: 15711347]

6. Brumovsky PR, Gebhart GF. Visceral organ cross-sensitization - an integrated perspective. Auton Neurosci. 2010; 153:106-15. [PubMed: 19679518]

7. Longstreth GF. Irritable bowel syndrome and chronic pelvic pain. Obstet Gynecol Surv. 1994; 49:505-507. [PubMed: 7936502]

8. Alagiri M, Chottiner S, Ratner V, Slade D, Hanno PM. Interstitial cystitis: unexplained associations with other chronic disease and pain syndromes. Urology. 1997; 49:52-57. [PubMed: 9146002]

9. Miranda A, Peles S, Shaker R, Rudolph C, Sengupta JN. Neonatal nociceptive somatic stimulation differentially modifies the activity of spinal neurons in rats and results in altered somatic and visceral sensation. J Physiol. 2006; 572:775-87. [PubMed: 16513666]

10. Coutinho SV, Meller ST, Gebhart GF. Intracolonic zymosan produces visceral hyperalgesia in the rat that is mediated by spinal NMDA and non-NMDA receptors. Brain Res. 1996; 736:7-15. [PubMed: 8930303]

11. Ide Y, Maehara Y, Tsukahara S, Kitahata LM, Collins JG. The effects of an intrathecal NMDA antagonist (AP5) on the behavioral changes induced by colorectal inflammation with turpentine in rats. Life Sci. 1997; 60:1359-1363. [PubMed: 9096256]

12. Kolhekar R, Gebhart GF. NMDA and quisqualate modulation of visceral nociception in the rat. Brain Res. 1994; 651:215-226. [PubMed: 7922569]

13. Kolhekar R, Gebhart GF. Modulation of spinal visceral nociceptive transmission by NMDA receptor activation in the rat. J Neurophysiol. 1996; 75:2344-2353. [PubMed: 8793747]

14. Zhou Q, Price DD, Caudle RM, Verne GN. Spinal NMDA NR1 subunit expression following transient TNBS colitis. Brain Res. 2009; 1279:109-20. [PubMed: 19406112]

15. Randich A, Meller ST, Gebhart GF. Responses of primary afferents and spinal dorsal horn neurons to thermal and mechanical stimuli before and during zymosan-induced inflammation of the rat hindpaw. Brain Res. 1997; 24(772):135-48. [PubMed: 9406965]

16. Randich A, Uzzell T, Cannon R, Ness TJ. Inflammation and enhanced nociceptive responses to bladder distension produced by intravesical zymosan in the rat. BMC Urol. 2006; 6:2. [PubMed: 16469099]

17. Randich A, Uzzell T, DeBerry JJ, Ness TJ. Neonatal urinary bladder inflammation produces adult bladder hypersensitivity. J Pain. 2006; 7:469-79. [PubMed: 16814686] 
18. Ustinova EE, Fraser MO, Pezzone MA. Colonic irritation in the rat sensitizes urinary bladder afferents to mechanical and chemical stimuli: an afferent origin of pelvic organ cross-sensitization. Am J Physiol Renal Physiol. 2006; 290:F1478-87. [PubMed: 16403832]

19. Liang R, Ustinova EE, Patnam R, Fraser MO, Gutkin DW, Pezzone MA. Enhanced expression of mast cell growth factor and mast cell activation in the bladder following the resolution of trinitrobenzenesulfonic acid (TNBS) colitis in female rats. Neurourol Urodyn. 2007; 26:887-93. [PubMed: 17385238]

20. Pezzone MA, Liang R, Fraser MO. A model of neural cross-talk and irritation in the pelvis: implications for the overlap of chronic pelvic pain disorders. Gastroenterology. 2005; 128:195364. [PubMed: 15940629]

21. Christianson JA, Liang R, Ustinova EE, Davis BM, Fraser MO, Pezzone MA. Convergence of bladder and colon sensory innervation occurs at the primary afferent level. Pain. 2007; 128:235243. [PubMed: 17070995]

22. Miranda A, Peles S, Rudolph C, Shaker R, Sengupta JN. Altered visceral sensation in response to somatic pain in the rat. Gastroenterology. 2004; 126:1082-9. [PubMed: 15057747]

23. Goldman JM, Murr AS, Cooper RL. The rodent estrous cycle: characterization of vaginal cytology and its utility in toxicological studies. Birth Defects Res B Dev Reprod Toxicol. 2007; 80:84-97. Review. [PubMed: 17342777]

24. Spanos C, Pang X, Ligris K, Letourneau R, Alferes L, Alexacos N, Sant GR, Theoharides TC. Stress-induced bladder mast cell activation: implications for interstitial cystitis. J Urol. 1997; 157:669-72. [PubMed: 8996395]

25. Sengupta JN, Gebhart GF. Mechanosensitive properties of pelvic nerve afferent fibers innervating the urinary bladder of the rat. J Neurophysiol. 1994; 72:2420-30. [PubMed: 7884468]

26. Banerjee B, Medda BK, Zheng Y, Miller H, Miranda A, Sengupta JN, Shaker R. Alterations in Nmethyl-D-aspartate receptor subunits in primary sensory neurons following acid-induced esophagitis in cats. Am J Physiol Gastrointest Liver Physiol. 2009; 296:66-77.

27. Ji Y, Tang B, Traub RJ. The visceromotor response to colorectal distention fluctuates with the estrous cycle in rats. Neuroscience. 2008; 154:1562-7. [PubMed: 18550290]

28. Ustinova EE, Gutkin DW, Pezzone MA. Sensitization of pelvic nerve afferents and mast cell infiltration in the urinary bladder following chronic colonic irritation is mediated by neuropeptides. Am J Physiol. 2007; 292:F123-130.

29. DeBerry J, Randich A, Shaffer AD, Robbins MT, Ness TJ. Neonatal bladder inflammation produces functional changes and alters neuropeptide content in bladders of adult female rats. J Pain. 2010; 11:247-55. [PubMed: 19945355]

30. Winnard KP, Dmitrieva N, Berkley KJ. Cross-organ interactions between reproductive, gastrointestinal, and urinary tracts: modulation by estrous stage and involvement of the hypogastric nerve. Am J Physiol Regul Integr Comp Physiol. 2006; 291:592-601.

31. Keast JR, de Groat WC, Keast JR, De Groat WC. Segmental distribution and peptide content of primary afferent neurons innervating the urogenital organs and colon of male rats. J Comp Neurol. 1992; 319:615-23. [PubMed: 1619047]

32. Ness TJ, Randich A. Neonatal bladder inflammation alters activity of adult rat spinal visceral nociceptive neurons. Neurosci Lett. 2010; 472:210-4. [PubMed: 20149841]

33. DeBerry J, Ness TJ, Robbins MT, Randich A. Inflammation-induced enhancement of the visceromotor reflex to urinary bladder distention: modulation by endogenous opioids and the effects of early-in-life experience with bladder inflammation. J Pain. 2007; 8:914-23. [PubMed: 17704007]

34. Hathway GJ, Koch S, Low L, Fitzgerald M. The changing balance of brainstem-spinal cord modulation of pain processing over the first weeks of rat postnatal life. J Physiol. 2009; 587:292735. [PubMed: 19403624]

35. Qin C, Foreman RD. Viscerovisceral convergence of urinary bladder and colorectal inputs to lumbosacral spinal neurons in rats. Neuroreport. 2004; 15:467-71. [PubMed: 15094505]

36. Al-Chaer ED, Kawasaki M, Pasricha PJ. A new model of chronic visceral hypersensitivity in adult rats induced by colon irritation during postnatal development. Gastroenterology. 2000; 119:127685. [PubMed: 11054385] 
37. Kashiwagi K, Pahk AJ, Masuko T, Igarashi K, Williams K. Block and modulation of N-methyl-Daspartate receptors by polyamines and protons: role of amino acid residues in the transmembrane and pore-forming regions of NR1 and NR2 subunits. Mol Pharmacol. 1997; 52:701-13. [PubMed: 9380034]

38. Zheng X, Zhang L, Durand GM, Bennett MV, Zukin RS. Mutagenesis rescues spermine and Zn2+ potentiation of recombinant NMDA receptors. Neuron. 1994; 12:811-8. [PubMed: 8161453]

39. Zhou Q, Price DD, Caudle RM, Verne GN. Visceral and somatic hypersensitivity in a subset of rats following TNBS-induced colitis. Pain. 2008; 134:9-15. [PubMed: 17481818] 


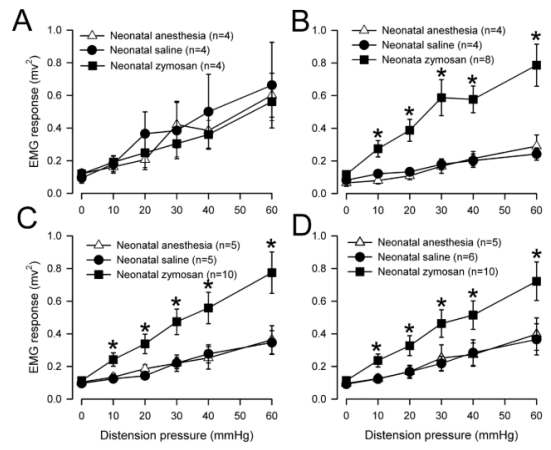

Figure 1.

VMR at different phases of the estrous cycle in adult female rats following neonatal intervention with intravesical zymosan, saline or anesthesia only. (A) In the proestrus phase, there was no difference in VMR between the groups. Conversely, the zymosan rats showed a significantly higher response to CRD in the diestrus (B) and estrus (C) phase at all distension pressures compared to saline and anesthesia control. (D) When all phases are combined, the mean VMR of the zymosan group was significantly higher in response to CRD at all distention pressures compared to the saline and anesthesia control. $\left({ }^{*} \mathrm{p}<0.05\right.$ vs. saline and anesthesia control). 

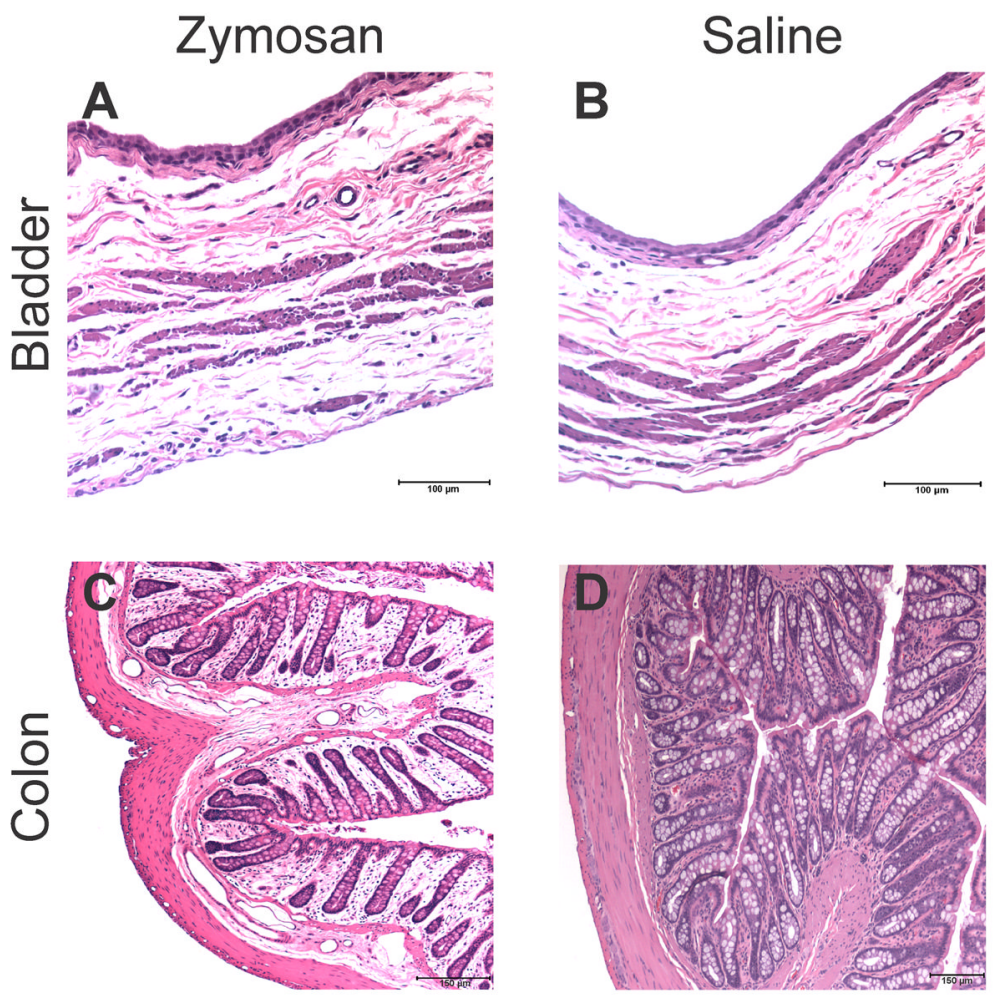

Figure 2.

Hematoxylin and eosin staining of adult bladder and distal colon tissue from neonatal zymosan and saline rats. Staining did not reveal any damage to the epithelium or significant polymophonuclear (PMN) cell infiltration in the bladder from either the zymosan (A) or saline treated rats (B). Similarly, there was no significant epithelial damage or inflammatory infiltrates in the colon of adult rats in either the neonatal zymosan group (C) or saline control (D). Scale bar=100 $\mu \mathrm{m}$ for figures (A) and (B), and $150 \mu \mathrm{m}$ for (C) and (D). 

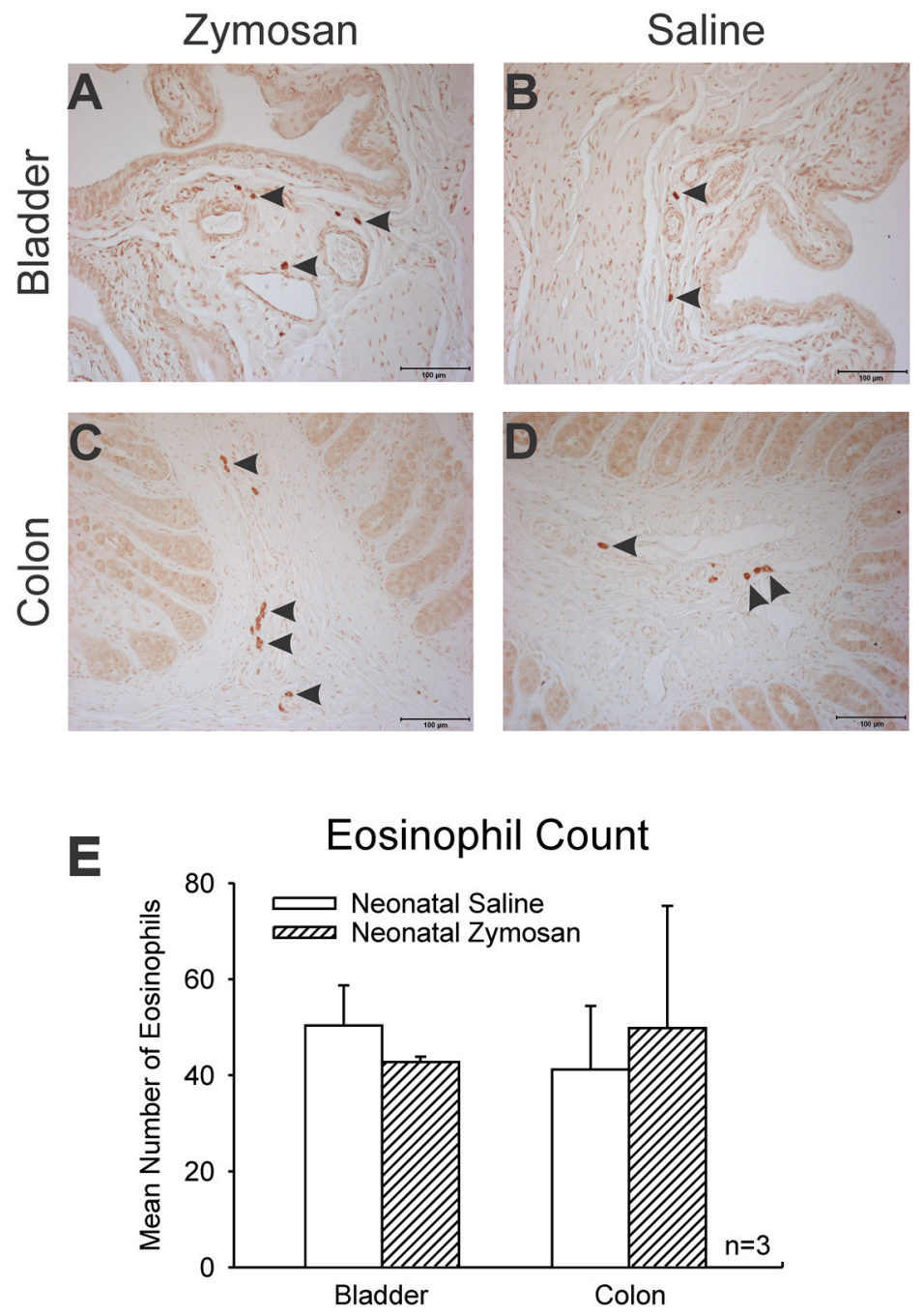

Figure 3.

Photomicrographs and quantitative analysis of eosinophils from the urinary bladder and colon tissue. Eosinophil infiltration (arrows) was determined by 3,3'-diaminobenzidine tetrahydrochloride (DAB) staining of cyanide-resistant EPO. Total counts were quantified from the bladder in adult rats with neonatal intravesical zymosan (A) or saline control (B). Similar quantification was performed between groups from colon tissue (C) and (D). Quantitative analysis of total eosinophil counts from colon or bladder did not show any significant difference between neonatal zymosan and saline control rats $(E)(p>0.05)$. Scale bar $=100 \mu \mathrm{m}$. 

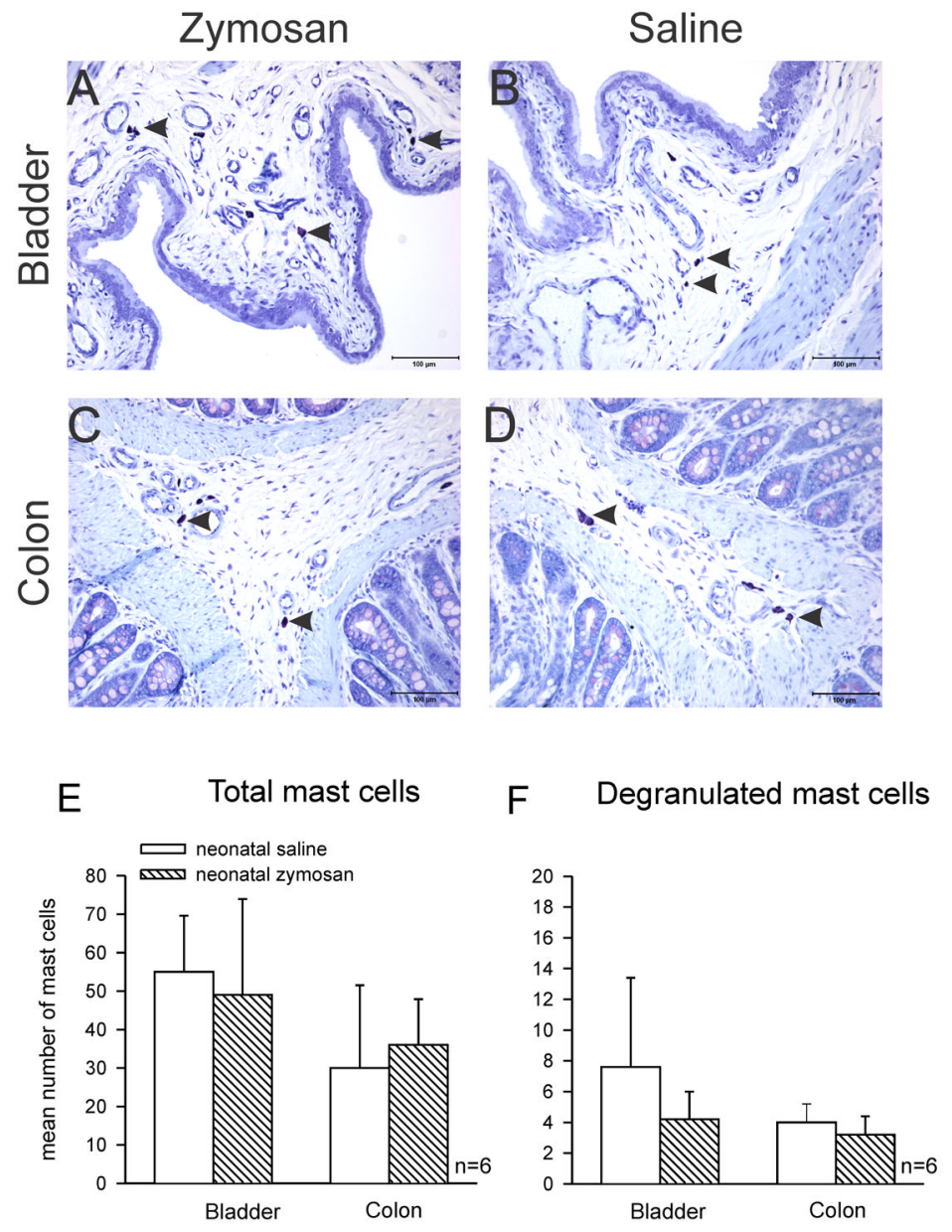

Figure 4.

Photomicrographs and quantitative analysis of mast cells from urinary bladder and colon tissue. Sections were stained with toluidine blue and total mast cells (arrows) as well as degranulated mast cell density was evaluated. Total counts and number of activated mast cells were quantified from the bladder in adult rats with neonatal intravesical zymosan (A) or saline control (B). Similar quantification was performed between groups from colon tissue (C) and (D). Quantitative analysis of total mast cells from colon or bladder (E) did not show any significant difference between neonatal zymosan and saline control. Similarly, the number of degranulated mast cells in the bladder and colon did not differ between groups (F) $(\mathrm{p}>0.05)$. Scale bar $=100 \mu \mathrm{m}$. 


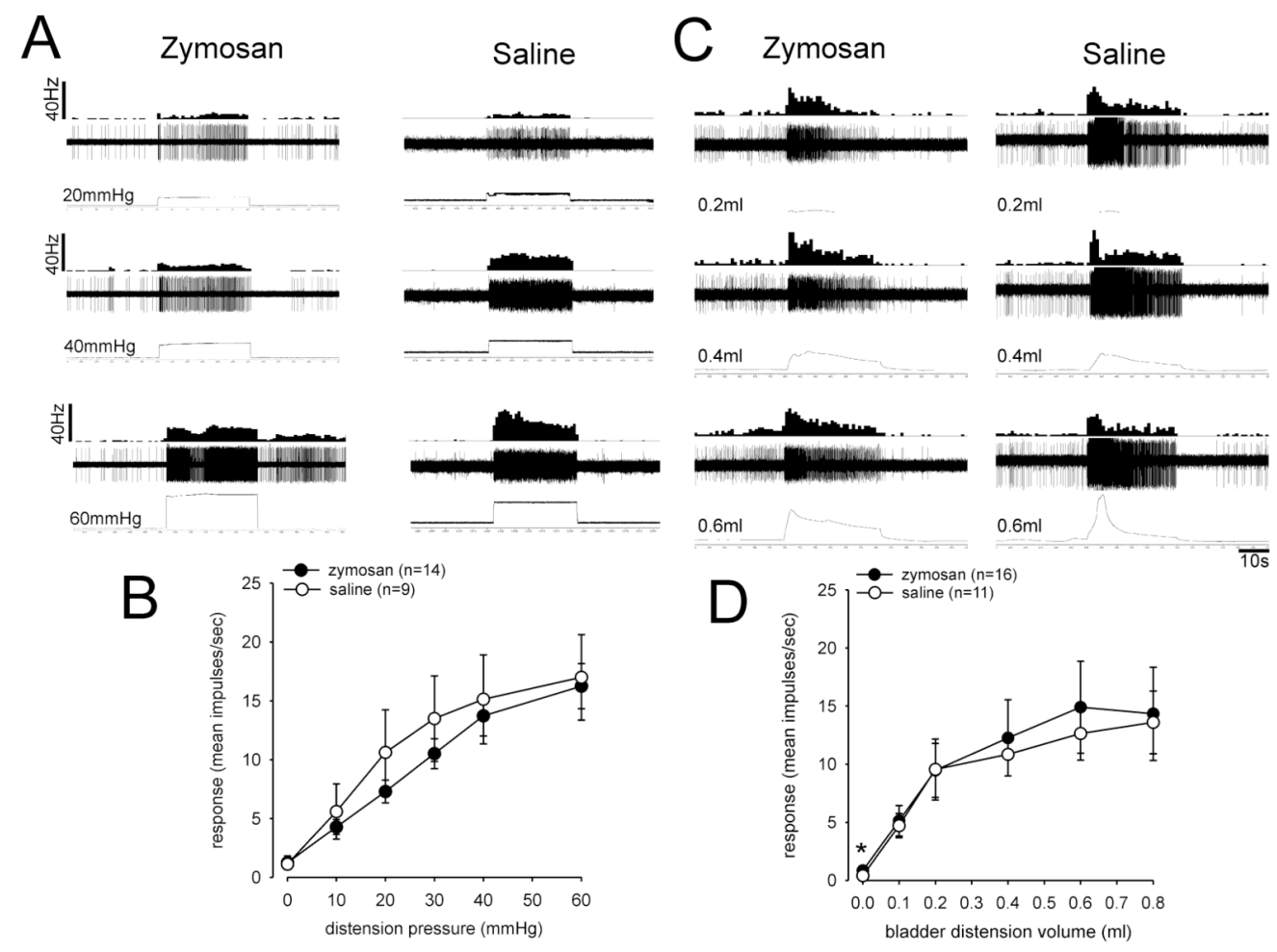

Figure 5.

(A): Illustrates an example of the mechanosensitivity of a CRD-sensitive PNA fiber to graded CRD from adult rats that received either intravesical zymosan or saline during the neonatal period. In all panels, the top tracing shows the response to CRD represented as a frequency histogram (1s binwidth), the middle trace is the neuron's action potentials and the bottom trace is the distension pressure. (B) The mean SRF of CRD-sensitive pelvic nerve afferent fibers showed that there was no difference in spontaneous firing or response to distension between groups ( $* p>0.05$ versus neonatal saline control). (C) Illustrates an example of the mechanosensitivity of a UBD-sensitive PNA fiber to bladder distension from adult rats that received either intravesical zymosan or saline during the neonatal period. (D) The mean SRF of UBD-sensitive PNA fibers showed that there was no difference in response to distension between groups ( $p>0.05$ versus neonatal saline control). However, the spontaneous firing of PNA fibers from zymosan treated rats was higher than saline control $(* \mathrm{p}=0.01)$. 


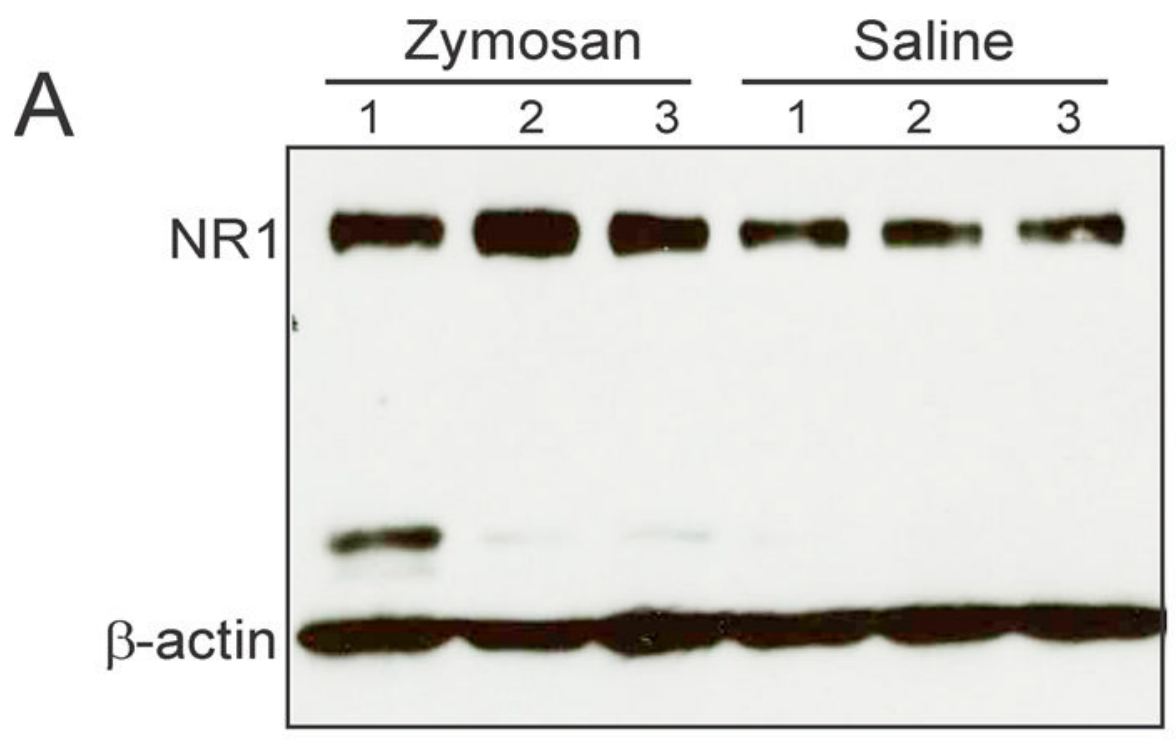

B

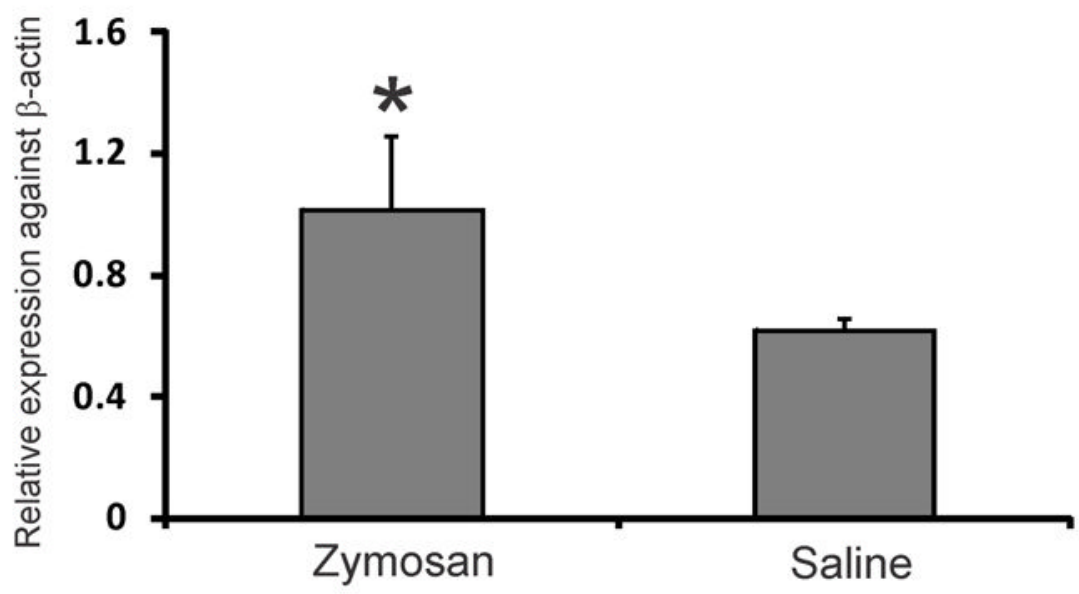

Figure 6.

Effect of neonatal intravesical zymosan on the polypeptide level of the NR1 subunit. A: Representative Western blot showing immunoreactivity in the lumbos-sacral spinal cord extracts from adult rats that received intravesical zymosan or saline during the neonatal period. The intensity of NR1 immunoreactivity for different tissues was normalized against the intensity of $\beta$-actin expression. B: The relative expression of NR1 was significantly higher in zymosan treated rats compared to saline treated rats $(* \mathrm{P}<0.05)$. 